\section{Relação entre o estado de humor e os comportamentos alimentares de risco para os transtornos alimentares em adolescentes}

\author{
Relationship between mood and risk eating \\ behaviors to eating disorders in adolescents
}

Leonardo de Sousa Fortes', Flávia Marcele Cipriani², Santiago Tavares Paes³, Fernanda Dias Coelho33, Maria Elisa Caputo Ferreira ${ }^{4}$

\section{RESUMO}

Objetivo: Analisar a relação entre o estado de humor e os comportamentos alimentares de risco para os transtornos alimentares (TA) em adolescentes do sexo feminino. Métodos: Participaram 397 adolescentes com idade entre 12 e 17 anos. Utilizaram-se as subescalas do Eating Attitudes Test (EAT-26) para avaliar os comportamentos alimentares de risco para os TA. Utilizou-se a Escala de Humor de Brunel (BRUMS) para avaliar o estado humor negativo (ansiedade, tensão, depressão, confusão mental, raiva e fadiga). Conduziram-se a Regressão Logística Binária e a Análise Multivariada de Covariância para analisar os dados. Resultados: Evidenciou-se associação estatisticamente significativa entre o estado de humor negativo e os comportamentos alimentares de risco para os TA $\left(X^{2}=25,71\right.$; Wald $=31,92$; $p=0,001)$. Identificou-se diferença de escore na subescala Dieta entre as adolescentes com alto e baixo estado de humor negativo $[F(1,396)=13,40 ; p=0,04]$, fato que não foi encontrado para as subescalas Bulimia e Preocupação com Alimentos $[F(1,396)=1,69 ; p=0,09]$ e Autocontrole Oral $[F(1,396)=0,95 ; p=0,17]$. Conclusão: Os comportamentos alimentares de risco para os TA estiveram relacionados ao estado de humor negativo.

\section{ABSTRACT}

Objective: To analyze the relationship between mood and risk eating behaviors to eating disorders (ED) in female adolescents. Methods: Three hundred and ninety-seven adolescents aged between 12 and 17 years participated. We used the subscales of the Eating Attitudes Test (EAT-26) to assess the risk eating behaviors to ED. We used the Brunel Mood Scale (BRUMS) to assess negative mood (anxiety, strain, depression, mental confusion, anger and fatigue). Led to Binary Logistic Regression and Multivariate Analysis of Covariance to analyze the data. Results: We revealed a statistically significant association between negative mood and risk eating behaviors to $E D\left(X^{2}=25.71\right.$; Wald $\left.=31.92 ; p=0.001\right)$. We identified differences in Diet subscale scores of adolescents with high and low negative $\operatorname{mood}[F(1,396)=13.40 ; p=0.04]$, which was not found for the Bulimia and Food Preoccupation subscale $[F(1,396)=1.69 ; p=0.09]$ and Selfcontrol Oral $[F(1,396)=0.95 ; p=0.17]$. Conclusion: The risk eating behaviors to ED were related to negative mood.

1 Universidade Federal de Pernambuco (UFPE), Núcleo de Educação Física e Ciências do Esporte, Programa de Pós-Graduação em Educação Física; Conselho Nacional de Desenvolvimento Científico e Tecnológico (CNPq), Grupo de Pesquisa "Psicologia Aplicada ao Esporte e Exercício".

Recebido em $5 / 9 / 2013$

Aprovado em $8 / 12 / 2014$

DOI: 10.1590/0047-2085000000117
2 Universidade Federal de Juiz de Fora (UFJF), Psicologia; CNPq, Grupo de Pesquisa "Corpo e Diversidade Humana".

3 UFJF, Educação Física; CNPq, Grupo de Pesquisa "Corpo e Diversidade Humana".

4 UFJF, Departamento de Fundamentos da Educação Física; CNPq, Grupo de Pesquisa "Corpo e Diversidade Humana".

Endereço para correspondência: Leonardo de Sousa Fortes

Rua Clóvis Beviláqua, 163/1003, Madalena

50710-330 - Recife, PE, Brasil

E-mail: leodesousafortes@hotmail.com 


\section{INTRODUÇÃO}

A restrição alimentar por longos períodos, a autoindução de vômitos, a compulsão alimentar e o uso de laxantes/diuréticos para o emagrecimento são classificados como comportamentos alimentares de risco para os transtornos alimentares (TA $)^{1,2}$. Tais comportamentos fazem parte dos critérios diagnósticos para a anorexia e a bulimia nervosa ${ }^{3}$. Evidências indicam prevalência de comportamentos alimentares de risco para os TA de aproximadamente 20\% no público adolescente ${ }^{4,5}$. Mais especificamente, a prevalência no sexo feminino parece ser ainda maior ${ }^{6,7}$.

A etiologia dos comportamentos alimentares de risco para os TA é multifatorial ${ }^{8,9}$. Diversos fatores estão associados ao desencadeamento desses comportamentos, tais como: autoestima, perfeccionismo, tecido adiposo, insatisfação corporal, sexo, faixa etária, entre outros ${ }^{10}$. Recentemente, Gonçalves et al. ${ }^{3}$ destacaram a possibilidade de os comportamentos alimentares de risco para os TA estarem associados ao estado de humor, compreendido como um conjunto de sentimentos positivos e negativos persistentes no momento e que variam em intensidade e duração' ${ }^{11}$.

Segundo Farias e Cordeiro'2, o estado de humor no sexo feminino pode ser afetado negativamente em função das mensagens subliminares midiáticas que exaltam ideais estéticos, do baixo rendimento escolar e das discussões fervorosas com familiares ou amigas. Considerando a possível relação entre o estado de humor e os comportamentos alimentares de risco para os $T^{3,13-16}$, as situações estressoras mencionadas acima podem predispor as adolescentes à adoção de comportamentos alimentares de risco para os TA. Acredita-se que esses comportamentos possam ser utilizados como alternativa para reduzir a magnitude dos sentimentos negativos.

Embora a literatura científica aponte relação do estado de humor com os comportamentos alimentares de risco para os TA ${ }^{3,12-16}$, nenhuma investigação científica com adolescentes brasileiras foi identificada até o momento. Diante do exposto, o objetivo do presente estudo foi analisar a relação entre o estado de humor e os comportamentos alimentares de risco para os TA em adolescentes do sexo feminino.

\section{MÉTODOS}

\section{Participantes}

Trata-se de estudo transversal, de base escolar, realizado nos anos de 2012 e 2013 na cidade de Juiz de Fora/MG, com adolescentes do sexo feminino na faixa etária entre 12 e 17 anos.

Segundo informações da Secretaria de Educação de Juiz de Fora, a população de adolescentes do sexo feminino com idade entre 12 e 17 anos, matriculada nas escolas do município em 2012 e 2013, era, respectivamente, de apro- ximadamente 41.000 e 41.200 jovens. Optou-se por inserir no cálculo amostral o maior valor populacional (41.200 jovens). Desse modo, realizou-se cálculo amostral utilizando os seguintes critérios, seguindo as recomendações de Alves et al. ${ }^{17}$ : prevalência de $20 \%$ para os comportamentos de risco para os TA de acordo com os achados de Fortes et al. ${ }^{5}$, 95\% de confiança e $5 \%$ de erro amostral, e ao total foi acrescido o valor de 20\% para possíveis perdas, totalizando 298 escolares que deveriam ser avaliadas para obter amostra que fosse representativa da população. O cálculo amostral foi procedido no programa Epi-Info (versão 3.5).

Foram incluídas na pesquisa somente as adolescentes que apresentaram o Termo de Consentimento Livre e Esclarecido (TCLE) assinado pelo responsável e que estavam regularmente matriculadas no ensino fundamental ou médio na cidade de Juiz de Fora/MG, no ano de 2012 ou 2013. Participaram do estudo 439 meninas; dessas, 42 foram excluídas por não responderem aos questionários por completo ou por não participarem das avaliações antropométricas, totalizando uma amostra final de 397 adolescentes.

\section{Instrumentos}

Para avaliar os comportamentos alimentares de risco para os TA, foi aplicado o Eating Attitudes Test ${ }^{18}$ (EAT-26) em sua versão validada para o sexo feminino por Bighetti et al. ${ }^{19}$, apresentando consistência interna igual a 0,82. Para o presente estudo, o instrumento apontou boa consistência interna, representada por $a=0,85$. O questionário é composto por 26 questões distribuídas em três fatores: 1) dieta - diz respeito à recusa patológica aos alimentos com alto teor calórico e à preocupação com a aparência física; 2) bulimia e preocupação com os alimentos - refere-se a episódios de compulsão alimentar, seguidos por comportamentos purgativos para perda/controle de peso corporal; e 3) autocontrole oral - reflete o autocontrole em relação à comida e avalia as forças ambientais e sociais estimulantes à ingestão alimentar. O escore final do EAT-26 é calculado pela soma de seus itens, e o ponto de corte para comportamento alimentar de risco para TA é 21, ou seja, pontuação menor que 21 indica comportamento desfavorável e pontuação maior ou igual a 21 representa atitudes favoráveis ao desenvolvimento de TA.

O estado de humor foi avaliado pela Escala de Humor de Brune ${ }^{20}$ (BRUMS) em sua versão validada para adolescentes ${ }^{11}$. Embora Rohlfs et al. "1" não tenham avaliado a consistência interna da BRUMS, para a presente amostra foi encontrado valor de alpha de Cronbach de 0,86, o qual representa boa consistência interna. A BRUMS contém 24 indicadores simples de humor, distribuídos em seis subescalas: tensão $(T)$, depressão $(D)$, raiva $(R)$, vigor $(V)$, fadiga $(F)$ e confusão mental (CM). Cada subescala contém quatro itens em escala do tipo Likert ( $\mathrm{Nada}=0 ; \mathrm{Um}$ pouco $=1$; Moderadamente $=2$; Bastante $=3$; Extremamente $=4$ ) e o escore pode variar de 0 a 16. As subescalas T, D, R, F e CM são consideradas os fatores 
negativos do humor, sendo o $\mathrm{V}$ considerado o fator positivo. O distúrbio total de humor (DTH) é calculado pela soma dos fatores negativos, subtraindo o escore do fator positivo. Ao resultado final do DTH, foram somados 100 para que não houvesse resultados negativos. Utilizou-se a média $(109,38)$ da BRUMS para classificar adolescentes com alto $(\geq 109,38)$ e baixo DTH $(<109,38)$.

Para avaliar a insatisfação corporal, foi aplicado o Body Shape Questionnaire ${ }^{21}$ (BSQ) em sua versão validada para a população de adolescentes brasileiros ${ }^{22}$. $O$ instrumento apresenta boa consistência interna [alpha de Cronbach (a) $=0,96]$ e coeficiente de correlação entre os escores do teste-reteste significativo $(0,89$ para meninas). Para amostra do presente estudo, identificou-se valor de $a=0,96$, demonstrando boa consistência do instrumento. O questionário autoavaliativo é composto por 34 perguntas em escala tipo Likert, relacionadas à preocupação que a jovem apresenta com o seu peso e com a sua aparência física. A avaliada aponta com que frequência, nas últimas quatro semanas, vivenciou os eventos propostos pelas alternativas e o escore final é dado pela soma total dos itens; quanto maior o escore, maior a insatisfação com o corpo. A classificação dos resultados do BSQ para adolescentes é dividida em quatro níveis de insatisfação corporal, sendo: pontuação abaixo de 80 indica ausência de insatisfação; entre 80 e 110, insatisfação leve; entre 110 e 140, insatisfação moderada; e igual ou acima de 140, grave insatisfação corporal. Em razão de algumas evidências apontarem a influência da insatisfação corporal sobre o comportamento alimentar de risco para os TA ${ }^{4,6}$, os escores do BSQ foram inseridos como covariáveis em algumas análises estatísticas da presente investigação.

Para o cálculo do percentual de gordura, foi utilizado o protocolo para adolescentes desenvolvido por Slaughter et al.23. Foram aferidas as dobras cutâneas tricipital e subescapular, de acordo com as padronizações determinadas pela International Society for Advancement for Kinanthropometry ${ }^{24}$, usando-se um compasso (adipômetro) cientííco da marca Lange ${ }^{\circledast}$ (Cambridge Scientific Industries Inc.), com precisão de $1 \mathrm{~mm}$. Em virtude de os achados de algumas investigações indicarem influência da gordura corporal sobre o comportamento alimentar de risco para $\mathrm{TA}^{5,6}, \mathrm{o}$ percentual de gordura foi inserido como covariável em algumas análises estatísticas no presente estudo.

\section{Procedimentos}

Os diretores de 10 escolas (cinco privadas e cinco públicas) foram convidados a participar da pesquisa, sendo informados sobre os objetivos e procedimentos. No entanto, somente seis deles (quatro de escolas privadas e dois de escolas públicas) concordaram em liberar as alunas para a participação nas coletas. Após a autorização das direções das escolas, foram realizadas reuniões com cada uma das turmas a fim de explicar os objetivos e procedimentos necessários para inclusão das escolares no estudo. Foi entregue o TCLE às adolescentes, pedindo-lhes que devolvessem devidamente assinados pelos responsáveis na semana seguinte, em caso de assentimento de sua participação voluntária.

A pesquisa foi realizada em dois momentos. Na primeira etapa as alunas responderam aos instrumentos (EAT-26, BRUMS e BSQ). Essa etapa foi realizada em grupo, por um único pesquisador, que padronizou as explicações verbais. Por conseguinte, após o preenchimento dos questionários, as alunas foram encaminhadas individualmente para aferição das medidas antropométricas (dobras cutâneas).

Este estudo obteve aprovação do Comitê de Ética e Pesquisa em Seres Humanos da Faculdade de Filosofia, Ciências e Letras da Universidade de São Paulo (Parecer no 109.971), de acordo com a Lei no 196/96 do Conselho Nacional de Saúde.

\section{Análise dos dados}

O teste Kolmogorov-Smirnov foi aplicado para avaliar a distribuição dos escores do EAT-26. Em razão da não violação paramétrica, utilizaram-se medidas de tendência central (média) e dispersão (mínimo, máximo e desvio-padrão) para descrever as variáveis da pesquisa (EAT-26, BRUMS, BSQ, idade e percentual de gordura). Utilizou-se a média $(109,38)$ da BRUMS para classificar adolescentes com alto $(\geq 109,38)$ e baixo DTH (< 109,38). Conduziu-se a Regressão Logística Binária para associar as classificações dicotômicas do EAT-26 com as da BRUMS. Ademais, a análise multivariada de covariância (MANCOVA), utilizando o BSQ e o percentual de gordura como covariáveis, foi aplicada para comparar os escores das subescalas do EAT-26 em função dos grupos estabelecidos a partir da média da BRUMS. O post hoc de Bonferroni foi utilizado para identificar a localização das diferenças estatísticas. Todos os dados foram tratados no software SPSS 20.0, adotando-se nível de significância de 5\%.

\section{RESULTADOS}

Os valores descritivos das variáveis analisadas estão apresentados na tabela 1. Os achados referentes à aplicação do EAT-26 evidenciaram que 23,3\% das adolescentes demonstraram comportamento alimentar de risco para os TA. Em adição, 40,6\% da amostra indicaram alto DTH (BRUMS $\geq$ 109,38). Além disso, os resultados apontaram que 33,9\% das adolescentes apresentaram algum nível de insatisfação corporal avaliado pelo BSQ.

O modelo de regressão logística indicou associação estatisticamente significativa entre o DTH e os comportamentos alimentares de risco para os TA $\left(X^{2}=25,71\right.$; Wald $=31,92 ; p=$ $0,001)$. Assim, as adolescentes com alto DTH demonstraram $46 \%$ de chances a mais para os comportamentos alimentares de risco para os TA quando comparadas às adolescentes com baixo DTH (Tabela 2). 
Tabela 1. Valores descritivos (mínimo, máximo, média e desvio-padrão) das variáveis do estudo - Juiz de Fora, 2012/2013

\begin{tabular}{lcccc}
\hline Variável & Mínimo & Máximo & Média & DP \\
\hline EAT-26 & 0 & 64 & 15,32 & 9,21 \\
BRUMS & 85 & 179 & 109,38 & 14,43 \\
BSQ & 34 & 192 & 70,99 & 36,85 \\
Idade (anos) & 12 & 17 & 13,74 & 1,70 \\
$\%$ G & 5,84 & 43,10 & 22,63 & 6,67 \\
\hline
\end{tabular}

DP: desvio-padrão; EAT-26: Eating Attitudes Test; BRUMS: Escala de Humor de Brunel; BSQ: Body Shape Questionnaire;\%G: percentual de gordura.

Tabela 2. Razão de prevalência para os comportamentos alimentares de risco para os transtornos alimentares em adolescentes do sexo feminino - Juiz de Fora, 2012/2013

\begin{tabular}{lccc}
\hline DTH & RP & IC (95\%) & pvalor \\
\hline Baixo & 1,00 & - & 0,001 \\
Alto & 1,46 & $1,13-5,59$ & \\
\hline
\end{tabular}

DTH: distúrbio total do humor; RP: razão de prevalência; IC: intervalo de confiança.

No que concerne às comparações das subescalas do EAT-26 em razão dos grupos de humor, alguns achados merecem destaque (Tabela 3): 1) identificou-se diferença de escore na subescala Dieta entre as adolescentes com alto e baixo DTH $[F(1,396)=13,40 ; p=0,04]$; e 2) as adolescentes com alto e baixo DTH demonstraram semelhanças nos escores das subescalas Bulimia e Preocupação com Alimentos $[F(1,396)=1,69 ; p=0,09)$ e Autocontrole Oral $[F(1,396)=$ $0,95 ; p=0,17]$. Salienta-se, sobretudo, que tanto os escores do $B S Q[F(1,396)=61,33 ; p=0,001]$ quanto o percentual de gordura $[F(1,396)=14,80 ; p=0,01]$ estiveram relacionados aos escores das subescalas do EAT-26.

Tabela 3. Comparação dos escores das subescalas do EAT-26 em função dos grupos de humor em adolescentes do sexo feminino - Juiz de Fora, 2012/2013

\begin{tabular}{|c|c|c|c|}
\hline \multicolumn{2}{|l|}{ Variável } & Grupo & p valor \\
\hline \multicolumn{2}{|c|}{ Baixo DTH (BRUMS < 109,38) } & \multicolumn{2}{|c|}{ Alto DTH (BRUMS $\geq 109,38)$} \\
\hline \multicolumn{4}{|c|}{ Média (EP) } \\
\hline Dieta & $7,17( \pm 0,75)$ & $9,35( \pm 0,91)$ & $\leq 0,04$ \\
\hline Bul e PA & $2,63( \pm 0,33)$ & $3,21( \pm 0,32)$ & $\leq 0,09$ \\
\hline AC Oral & $4,23( \pm 0,40)$ & $4,63( \pm 0,48)$ & $\leq 0,17$ \\
\hline
\end{tabular}

Bul e PA: Bulimia e Preocupação com Alimentos; AC: Autocontrole; BRUMS: Escala de Humor de Brunel; EP: erro-padrão; EAT-26: Eating Attitudes Test.

\section{DISCUSSÃO}

O presente estudo teve como principal premissa analisar a relação entre o estado de humor e os comportamentos alimentares de risco para os TA em adolescentes do sexo feminino. Segundo Gonçalves et al. ${ }^{3}$, o estado de humor negativo pode predispor meninas à adoção de comportamentos ali- mentares de risco para os TA. Nesse sentido, adolescentes com sentimentos de raiva, depressão, tensão e/ou fadiga podem buscar nos comportamentos alimentares de risco para os TA uma alternativa para melhorar o humor. Salienta-se, no entanto, que nenhuma investigação científica que tenha utilizado amostra de adolescentes brasileiras foi identificada até o momento.

Os achados demonstraram prevalência de 23,3\% para os comportamentos alimentares de risco para os TA. Outras investigações que também utilizaram o EAT-26 como instrumento avaliativo corroboram esses resultados ${ }^{4-6}$. De acordo com Rodgers et al. ${ }^{9}$, em razão da contradição apresentada pela mídia ao preconizar a magreza como sinônimo de felicidade/sucesso e ao mesmo tempo incentivar a ingestão de alimentos com alto teor calórico, algumas adolescentes optam por adotar os comportamentos alimentares de risco para os TA como medida para a redução do peso corporal. Assim, a prevalência desses comportamentos tem aumentado nos últimos anos entre as adolescentes ${ }^{7,8}$.

Evidenciou-se associação entre os comportamentos alimentares de risco para os TA e o DTH. Os resultados indicaram maiores chances de as adolescentes com alto DTH utilizarem os comportamentos alimentares de risco para os TA em comparação às adolescentes com baixo DTH. Dessa maneira, as meninas com sentimentos elevados de raiva, depressão, tensão e/ou fadiga usaram com maior frequência os comportamentos alimentares de risco para os TA, conforme Gonçalves et al. ${ }^{3}$ ressaltaram. Considerando que o estado de humor pode ser alterado em função da dieta adotada ${ }^{25}$, é possível que as adolescentes com alto DTH tenham utilizado os comportamentos compulsivos, purgativos ou a restrição alimentar com o intuito de reduzir a magnitude dos sentimentos humorais negativos. A investigação de Liao et al. ${ }^{16}$ corrobora a associação entre o estado de humor negativo (depressão e tensão) e os comportamentos alimentares de risco para os TA no sexo feminino. Esses autores evidenciaram que universitárias chinesas com sintomas de depressão e estado emocional de tensão estavam mais suscetíveis à adoção dos comportamentos alimentares de risco para os TA. Do mesmo modo, Allen et al. ${ }^{13}$ identificaram relação positiva entre o estado de humor negativo e os comportamentos alimentares de risco para os TA em mulheres australianas.

Cabe salientar que o humor pode sofrer variações negativas em virtude de situações cotidianas, tais como: discussões com amigos, baixo rendimento escolar, comentários depreciativos de amigos a respeito do peso corporal, brigas familiares, entre outras ${ }^{12}$. Logo, em decorrência das situações estressoras mencionadas, as adolescentes podem aumentar a suscetibilidade para o desencadeamento de comportamentos alimentares de risco para os TA.

Concernente à análise de MANCOVA, os achados apontaram maior frequência de restrição alimentar nas adolescen- 
tes com alto DTH quando comparadas às adolescentes com baixo DTH, o que corrobora outros achados ${ }^{13,15}$. Parece que o comportamento alimentar restritivo é comum em meninas com sentimentos de raiva, depressão e tensão ${ }^{25}$. Talvez, o humor negativo seja responsável por secretar neurotransmissores cerebrais, os quais podem estar associados à inibição do apetite. Vale salientar que os sentimentos de raiva, tensão e depressão são oriundos de situações estressantes vividas por adolescentes ${ }^{12}$. Desse modo, após episódios que gerem aumento do DTH, é possível que as adolescentes utilizem a restrição alimentar de forma inconsciente, o que, de certo modo, pode gerar problemas hormonais e atraso no crescimento físico ${ }^{26}$.

Por outro lado, os resultados da MANCOVA não indicaram diferença da frequência de comportamentos compulsivos e purgativos entre adolescentes com DTH diferente. A frequência de compulsão alimentar e/ou o uso de laxantes e diuréticos foi semelhante entre as adolescentes com alto e baixo DTH. Nesse sentido, o estado de humor negativo não foi responsável por induzir as adolescentes à adoção de comportamentos relacionados aos sintomas de bulimia. Em contrapartida, Silva et al. ${ }^{15}$ analisaram universitárias chilenas e evidenciaram relação positiva entre o estado de humor negativo e a patologia bulímica. Em adição, alguns autores ${ }^{25}$ argumentam que as adolescentes podem utilizar a voracidade alimentar como alternativa na diminuição da magnitude dos sentimentos humorais negativos. Segundo esses autores, a elevada ingestão de alimentos ricos em carboidratos (doces) é capaz de potencializar a secreção do neurotransmissor serotonina, o qual está associado aos sentimentos de felicidade ${ }^{26}$. Mais investigações são necessárias para esclarecer melhor esse tópico.

Os achados também não indicaram diferença de autocontrole em relação à comida e à percepção das forças ambientais e sociais estimulantes à ingestão alimentar entre adolescentes com alto e baixo DTH. De acordo com Farias e Cordeiro ${ }^{12}$, o estado de humor não é capaz de alterar a pressão percebida para a ingestão alimentar por pais e amigos em adolescentes do sexo feminino, o que explica tais resultados. As percepções ambientais para a alimentação parecem ser influenciadas principalmente por características pessoais como a personalidade ${ }^{27}$. Dessa forma, o estado de humor, caracterizado por sentimentos que oscilam em tempo e intensidade, parece não alterar a forma como a adolescente é influenciada em sua alimentação por pais e amigos. Todavia, outros pesquisadores ressaltam a possibilidade de os sentimentos de raiva, depressão e tensão gerarem maior percepção negativa de forças ambientais (pais, amigos e mídia) para a ingestão de alimentos $^{3}$. Salienta-se, sobretudo, que nenhuma evidência científica que pudesse suportar essa hipótese foi encontrada.

Embora essa investigação demonstre resultados inéditos, faz-se necessário mencionar algumas limitações. Uma delas foi utilizar os questionários como instrumentos princi- pais. Pesquisadores afirmam que os indivíduos podem não responder com fidedignidade a ferramentas autoaplicáveis ${ }^{1,9}$. Portanto, os resultados podem não refletir a realidade do contexto avaliado, visto que o resultado final é fruto de respostas subjetivas. Entretanto, pesquisadores destacam a relevância desses instrumentos, desde que tenham suas qualidades psicométricas asseguradas ${ }^{8}$. Outra limitação foi o delineamento do tipo transversal. Desse modo, não foi possível realizar inferência de causalidade. Isso significa que não é possível avaliar o grau de intensidade e a direção das associações encontradas entre o desfecho do estudo e as variáveis independentes. Acrescentando, a BRUMS20 não foi validada para a população-alvo deste estudo, o que representa uma limitação importante. Entretanto, a elevada consistência interna desse instrumento entre as adolescentes participantes desta investigação dá indícios de boas qualidades psicométricas da escala também entre essa população. Por fim, a não avaliação da etapa do ciclo menstrual e do uso de contraceptivo oral pode ser apontada como outra limitação da investigação, em razão de esses aspectos apresentarem relativa influência no estado de humor de adolescentes do sexo feminino. A despeito dessas limitações, acredita-se que a presente pesquisa mostra resultados importantes que merecem ser discutidos na literatura científica.

\section{CONCLUSÃO}

Os comportamentos alimentares de risco para os TA estiveram relacionados ao estado de humor. Os resultados permitem inferir que as adolescentes com alto DTH estão mais suscetíveis à adoção de comportamentos alimentares de risco para os TA. Todavia, tais achados devem ser interpretados com cautela por causa das limitações apresentadas pela presente investigação.

Recomenda-se a inserção de programas de acompanhamento psicológico e nutricional em escolas das redes pública e privada a fim de reduzir a prevalência de comportamentos alimentares de risco para os TA em adolescentes do sexo feminino. Além disso, sugere-se que sejam realizados estudos averiguando a relação de outros fatores com os comportamentos alimentares de risco para os TA em adolescentes, bem como observações que busquem investigar a direção dessas relações.

\section{CONTRIBUIÇÕES INDIVIDUAIS}

Leonardo de Sousa Fortes - Elaborou o projeto de pesquisa, coletou os dados e redigiu o artigo.

Flávia Marcele Cipriani - Auxiliou na coleta dos dados e revisou todo o português do artigo. 
Santiago Tavares Paes - Auxiliou na coleta dos dados e revisou a análise dos dados.

Fernanda Dias Coelho - Auxiliou na coleta dos dados e revisou o artigo.

Maria Elisa Caputo Ferreira - Orientou todo o trabalho e revisou o artigo.

\section{AGRADECIMENTOS}

Às adolescentes participantes deste estudo, pela colaboração. Ao Laboratório de Estudos do Corpo (UFJF), pela disponibilização dos instrumentais do estudo. Ao Conselho Nacional de Desenvolvimento Científico e Tecnológico (CNPq), pelo financiamento do projeto.

\section{REFERÊNCIAS}

1. de Souza Ferreira JE, da Veiga GV. Eating disorder risk behavior in Brazilian adolescents from low socio-economic level. Appetite. 2008;51(2):249-55.

2. Sampei MA, Singulem DM, Novo NF, Juliano Y, Colugnati FAB. Atitudes alimentares e imagem corporal em meninas adolescentes de ascendência nipônica e caucasiana em São Paulo (SP). J Pediatr (Rio J). 2009;85(2):122-8.

3. Gonçalves JA, Moreira EAM, Trindade EBSM, Fiates GMR. Transtornos alimentares na infância e na adolescência. Rev Paul Pediatr. 2013;31(1):96-103.

4. Fortes LS, Amaral ACS, Almeida SS, Ferreira MEC. Efeitos de variáveis psicológicas, morfológicas e sociodemográficas sobre o comportamento alimentar de adolescentes. Rev Paul Pediatr. 2013;31(2):182-8.

5. Fortes LS, Morgado FFR, Ferreira MEC. Fatores associados ao comportamento alimentar inadequado em adolescentes escolares. Rev Psiquiatr Clin. 2013;40(2):59-64.

6. Scherer FC, Martins CR, Pelegrini A, Matheus SC, Petroski EL. Imagem corporal em adolescentes: associação com a maturação sexual e sintomas de transtornos alimentares. J Bras Psiquiatr. 2010;59(3):198-202.

7. Martins CR, Pelegrini A, Matheus SC, Petroski EL. Insatisfação com a imagem corporal e relação com estado nutricional, adiposidade corporal e sintomas de anorexia e bulimia em adolescentes. Rev Psiquiatr Rio Gde Sul. 2010;32(1):19-23.

8. Flament MF, Hill EM, Buchholz A, Henderson K, Tasca GA, Goldfield G. Internalization of the thin and muscular body ideal and disordered eating in adolescence: the mediation effects of body esteem. Body Image. 2012;9(1):68-75.

9. Rodgers R, Chabrol H, Paxton SJ. An exploration of the tripartite influence model of body dissatisfaction and disordered eating among Australian and French college women. Body Image. 2011;8(3):208-15.
10. Leal GVS, Philippi ST, Polacow VO, Cordás TA, Alvarenga MS. 0 que é comportamento de risco para transtornos alimentares em adolescentes? J Bras Psiquiatr. 2013;62(1):62-75.

11. Rohlfs ICPM, Rotta TM, Luft CDB, Andrade A, Krebs RJ, Carvalho T. A Escala de Humor de Brunel (BRUMS): Instrumento para detecção precoce da síndrome do excesso de treinamento. Rev Bras Med Esporte. 2008;14(3):176-81.

12. Farias AC, Cordeiro ML. Mood disorders in children and adolescents: update for pediatricians. J Pediatr (Rio J). 2011:87(5):373-81.

13. Allen KL, McLean NJ, Byrne SM. Evaluation of a new measure of mood intolerance, the Tolerance of Mood States Scale (TOMS): psychometric properties and associations with eating disorder symptoms. Eat Behav. 2012;13(4):326-34.

14. Tchanturia K, Davies H, Harrison A, Fox JR, Treasure J, Schmidt U. Altered social hedonic processing in eating disorders. Int J Eat Disord. 2012;45(8):962-9.

15. Silva JR, Ortiz M, Quiñones Á, Vera-Villarroel P, Slachevsky A. Affective style and eating disorders: a field study. Eat Weight Disord. 2011;16(2):e73-80.

16. Liao Y, Knoesen NP, Castle DJ, Tang J, Deng Y, Bookun R, et al. Symptoms of disordered eating, body shape, and mood concerns in male and female Chinese medical students. Compr Psychiatry. 2010;51(5):516-23.

17. Alves E, Vasconcelos FAG, Calvo MCM, Neves J. Prevalence of symptoms of anorexia nervosa and dissatisfaction with body image among female adolescents in Florianópolis, Santa Catarina State, Brazil. Cad Saude Publica. 2008;24(3):503-12

18. Garner DM, Olmsted MP, Bohr Y, Garfinkel PE. The eating attitudes test: psychometric features and clinical correlates. Psychol Med. 1982;12(4):871-8.

19. Bighetti F, Santos (B, Santos JE, Ribeiro RPP. Tradução e avaliação do Eating Attitudes Test em adolescentes do sexo feminino de Ribeirão Preto, São Paulo. J Bras Psiquiatr. 2004;53(6):339-46.

20. Terry PC, Lane AM, Lane HJ, Keohane L. Development and validation of a mood measure for adolescents. J Sports Sci. 1999;17(11):861-72.

21. Cooper PJ, Taylor M, Cooper Z, Fairburn CG. The development and validation of the Body Shape Questionnaire. Int J Eat Disord. 1987;6(3):485-94.

22. Conti MA, Cordás TA, Latorre MRDO. Estudo de validade e confiabilidade da versão brasileira do Body Shape Questionnaire (BSQ) para adolescentes. Rev Bras Saúde Mater Infant. 2009;9(3):331-8.

23. Slaughter MH, Lohman TG, Boileau RA, Horswill CA, Stillman RJ, Van Loan MD, et al. Skinfold equations for estimation of body fatness in children and youth. Hum Biol. 1988:60(5):709-23.

24. ISAK - The Internacional Society for Advancement for Kineanthropometry. First printed. Australia: National Library of Australia; 2001.

25. Rouveix M, Bouget M, Pannafieux C, Champely S, Filaire E. Eating attitudes, body esteem, perfectionism and anxiety of judo athletes and nonathletes. Int I Sports Med. 2007;28(2):340-5.

26. Fortes LS, Almeida SS, Ferreira MEC. Maturation process, body dissatisfaction and inappropriate eating behavior in young athletes. Rev Nutr. 2012;25(5):575-86.

27. Filaire E, Rouveix M, Pannafieux C, Ferrand C. Eating atitudes, perfectionism and bodyesteem of elite male judoists and cyclists. J Sports Sci Med. 2007;6(1):50-7. 